\title{
Investigation of the mechanism of photochemically-induced lysozyme crystallization
}

Tetsuo Okutsu*, Tohru Taguchi, Jyunya Korenaga, Takashi Kuroiwa, Yu Ishikawa, Shiori Iizuka, Kaori Sugiyama, Hiroaki Horiuchi, Hiroshi Hiratsuka

Division of Molecular Science, Faculty of Science and Technology, Gunma University,

1-5-1 Tenjin-cho, Kiryu, Gunma 376-8515

Received: September, 2015; $\quad$ E-mail: okutsu@gunma-u.ac.jp

\begin{abstract}
This work examined the mechanism involved in photoinduced crystallization of a lysozyme, focusing on the structure of the photoproducts that lead to crystal growth. It was determined that dimers formed via linking of tryptophan (Trp) residues are preferentially produced when applying a high-excitation photon fluence. However, these dimers do not lead to crystal growth. A low fluence forms dimers via the combination of tyrosine (Tyr) residues, generating six types of Tyr-Tyr dimers. The dimer that originates from the linking of $\mathrm{Tyr}^{53}$ and $\mathrm{Tyr}^{53}$ has a configuration similar to that of the two adjacent, yet non-linked, lysozyme molecules in the crystal unit cell. The Tyr ${ }^{53-T y r}{ }^{53}$ dimer hence acts as a template for lysozyme crystal nucleation.
\end{abstract}

Key words: Photo-induced crystallization, lysozyme, template 


\section{Introduction}

The crystallization of proteins is an important technique in genome-based drug discovery and further developments in this technology are expected. At present, the primary means of protein structural elucidation is X-ray crystallographic analysis, and the number of proteins analyzed has increased dramatically since synchrotron radiation instrumentation has been applied for this purpose. The present bottleneck is in fact the rate at which new protein crystals can be produced.

High-throughput protein crystallization is performed by large-scale screening processes, and various physical and chemical studies have been performed with the aim of finding methods to induce crystal formation from solution. The laser-induced method of crystallization, both discovered and developed over the past ten years, is of particular interest [1-4], and the application of this technique to the crystallization of proteins is currently an active research area. As an example, a group at Osaka University has reported that the emergence of protein crystals was promoted by irradiation of a protein solution with a femtosecond laser [5-7]. The effectiveness of this method is believed to be due to the alignment of molecules by the strong electric field associated with the laser light or from local condensation of solute molecules under strong photon pressure [8,9]. Methods of using laserinduced photon pressure to collect molecules at the light condensing point are also a topic of research [10].

Our own group has previously observed the photochemically-induced crystallization of lysozymes $[11,12]$. Herein, we describe the mechanism that 
we have so far elucidated to explain why crystals are readily formed when proteins are irradiated by electromagnetic radiation with wavelengths in the UV-visible region of the spectrum [13-17].

Previous studies by Adachi et al. [5] and ourselves [14] have demonstrated the presence of covalently bonded protein dimers in irradiated solutions. In the initial stage of crystal growth, small clusters are thermodynamically unstable and cannot grow into bulk crystals even if the solution is supersaturated. However, in the case that nucleation is initiated from stable bimolecular precursors that cannot dissociate due to their covalent linkage, then critical nuclei can be easily formed.

Empirical studies have shown that the onset of photoinduced crystallization depends on the light source employed. The use of a highpower light source does not result in protein crystallization, whereas irradiation with a weak continuous wave (cw) light source does induce crystallization. On the basis of such results, we believe that the photon fluence per unit time is important to the induction of crystallization. The excitation photon fluence is also thought to affect the structure of the photoproduct. Thus, it is possible that dimers generated by excitation with a high-fluence light source may adopt structures that do not serve as nuclei, while dimers produced by irradiation with a low-fluence source will have a structure that readily grows into nuclei.

In this study, we examined the relationship between the structures of dimers that grow into crystal nuclei and the reaction mechanism involving intermediate radicals. We also assessed, at the amino acid level, whether or 
not templates are formed by the photochemical reaction of lysozymes in solution.

\section{Experimental}

Hen egg white lysozyme was purchased from Seikagaku (six times recrystallized, lot E02Z04) and was used without further purification. Sodium acetate, acetic acid and sodium chloride $(\mathrm{NaCl})$, all $\mathrm{GR}$ grade, were purchased from Wako Pure Chemicals. The sodium acetate and acetic acid were dissolved in ultrapure water (Milli-Q) to produce a buffer solution (NaAc buffer, $50 \mathrm{mM}, \mathrm{pH}$ 4.3). This solution was centrifuged and filtered through a $0.45 \mu \mathrm{m}$ single-use membrane (Millipore) prior to each experimental trial. Crystallization was performed under metastable conditions, typically with supersaturation $\left(\mathrm{C} / \mathrm{C}_{\mathrm{e}}=3\right)$ and employing the batch method. The protein crystallization in this work followed a wellknown procedure commonly used by biochemists and described in various textbooks $[18,19]$.

A Xe lamp (150 W, USHIO) was employed as a light source for the light-induced crystallization trials. For transient absorption measurements, a $\mathrm{Nd}^{3+}:$ YAG laser (Tokyo Instruments Lotis II, $266 \mathrm{~nm}$, pulse width $8 \mathrm{~ns}, 10$ Hz) was used as the excitation light source. Details of the experimental setup for the transient absorption experiments have been provided previously in the literature [12]. Steady-state emission was recorded on a HITACHI F4500 fluorescence spectrometer and absorption spectra were recorded with a HITACHI U3300 spectrophotometer. The number of photons 
emitted by the Xe lamp was measured at the irradiated position with a spectroradiometer (USHIO USR 30). 
3. Results and Discussion

3.1. Template formation in crystals and solutions

In this study, we first generated templates and conducted experiments to confirm the growth of crystal nuclei. Templates were obtained by irradiating protein crystals with light, assuming that dimers generated by the reaction between adjacent molecules in the crystal would act as templates for crystal formation. In contrast, photochemically-induced dimers resulting from reactions in solution can occur at various sites, and so the position of the amino acid that reacts is not limited. Therefore, not all the dimers in the solution will have a structure that functions as a template. For this reason, we predicted that the template generation efficiency would vary between cases in which dimers were generated by reactions in a crystal or in a solution.

Figure 1(a) shows the results obtained from a control trial in which we dissolved untreated crystals in a buffer solution, added a precipitant, and allowed the solution to stand for $24 \mathrm{~h}$. Crystals did not emerge from this solution because the concentration was low. Figure 1(b) presents an image from an experiment in which we irradiated a solution of the lysozyme and then added a precipitant. Approximately 20 crystals were formed in this solution. Finally, a trial was conducted in which crystals were first irradiated and then dissolved in the buffer along with the precipitant. The result is shown in Figure 1(c). The number of crystals generated in this case was approximately four times greater than the number obtained in the trial in which the protein solution was irradiated (Figure 1(b)). This result 
demonstrates that products generated in the solid state can serve as templates, and suggests that a greater number of templates suitable for crystal formation were present in the last trial. It is considered that the crystalline products have the same conformation as the two adjacent molecules in the initial dimer, and that these dimers thus grow to form nuclei that serve as templates in the supersaturated solution.

To confirm the product formation, we obtained SDS-PAGE data for the various solutions, with the results provided in Figure 2. The lysozyme dimer was used as a reference in these analyses. The non-irradiated solution generated only the monomer band, while dimer bands were produced from both the irradiated solution and the solution of pre-irradiated crystals of the lysozyme substrate.

\subsection{Photochemical reaction mechanism}

We subsequently examined the relationship between the structure of the reaction intermediates and the dimers generated by the photochemical reaction in solution. Figure 3(a) shows the transient absorption spectra obtained from the lysozyme. The component that absorbs in the range of 350-600 $\mathrm{nm}$ in this spectrum has been reported to be a reaction intermediate of the $\operatorname{Trp}^{62}$ residue [20,21], while the absorption from 600 to $700 \mathrm{~nm}$ is attributed to solvated electrons. Scheme 1(a) shows the initial stage of the photochemical reaction of the lysozyme. In this process, the lysozyme absorbs light and generates an excited state consisting of Trp residues. An excited triplet state is subsequently generated through 
intersystem crossing (ISC), which in turn produces the Trp residue radicals that serve as reaction intermediates. Transient absorption experiments reported in a previous study showed that these protein radicals can decay via both first-order and second-order reaction mechanisms [14]. The secondorder decay mechanism involves the combination of protein radicals, as shown in Scheme 1(b). One such dimer generated in this process is Trp ${ }^{62}$ $\operatorname{Trp}^{62}$ and the structure of this dimer is presented in Scheme 1(c).

Alternatively, the residual Trp radicals can also decay through a first-order process and the associated reaction mechanism is shown in Scheme 2, based on radiation chemistry studies [22]. This prior work demonstrated that bromine radicals generated by radiation can react with the lysozyme to generate reaction intermediate radicals that include Trp radicals. This reaction mechanism is illustrated in Scheme 2(a). These radicals abstract hydrogen atoms from neighboring Tyr residues in the molecule to generate intermediates based on residual Tyr radicals, as in Scheme 2(b). Overall, this reaction process involving the lysozyme molecule corresponds to a firstorder decay.

The second-order decay process to produce Trp-Trp dimers preferentially occurs at high radical concentrations, such as when the sample is excited with a high photon fluence. Conversely, the first-order process to produce Tyr-Tyr dimers is favored at low radical concentrations, such as when the sample is excited with a low photon fluence, as illustrated in Scheme 2(c). It should therefore be possible to switch between these two decay mechanisms by varying the excitation photon fluence. 
In this context, we decided to study the potential switching between second-order and first-order processes by variation of the photon fluence.

In the experiments reported herein, we varied the fluence by changing the pulse power of the YAG laser. Figure 3(b) presents the decay time profile for Trp radicals observed at $550 \mathrm{~nm}$ with the fluence of the excitation light decreased from 7 to $2 \mathrm{~mJ}$ pulse $\mathrm{s}^{-1}$. The initial decay up to $20 \mu$ s results from ISC and the population of the excited triplet state. Although the absorbance was attenuated at high fluence levels over the observed time interval, it was significantly less attenuated when applying a low fluence following promotion to the excited triplet state. The rate equation for the second-order reaction mechanism is $\mathrm{d}[\operatorname{Trp} \cdot] / \mathrm{d} t=-k_{2}[\operatorname{Trp} \cdot] 2$, which is equivalent to $1 /$ $[\operatorname{Trp} \cdot]=1 /[\operatorname{Trp} \cdot]_{t=0}+k t$. Thus, if a linear relationship is obtained from a plot of the reciprocals of the experimental results, this indicates attenuation via the second order process. The rate equation for attenuation in the firstorder process is $\mathrm{d}[\operatorname{Trp} \cdot] / \mathrm{d} t=-k_{1}[\operatorname{Trp} \cdot]$ or $\ln [\operatorname{Trp} \cdot]=-k_{1} t+\ln [\operatorname{Trp} \cdot]_{t=0}$. Therefore, if a logarithmic plot of the absorbance change shows a linear relationship, then the first-order reaction mechanism occurs. Figures 3(c) and $3(\mathrm{~d})$ show the reciprocal and logarithmic plots, respectively, of the radical attenuation. The reciprocal plot shown in Figure 3(c) is roughly linear at high fluence in the time domain following attenuation of the excited triplet state. This linearity demonstrates that the process in which dimers are generated by radical-radical combination is dominant at high radical concentrations. In contrast, the logarithmic plot shown in Figure 3(d) can be approximated by a straight line only under low fluence 
conditions. This result suggests a mechanism in which, at low radical concentrations, Trp radicals are formed by an initial reaction and are afterwards converted into other radicals within the protein molecules.

In this work, we conducted experiments to confirm whether or not photochemically generated dimers can function as templates for crystal growth. Furthermore, we examined the effects of the differences in mechanism and the associated alternate dimer formations on crystal growth. In crystallization experiments, we varied the radiation fluence by expanding the beam of irradiating light with a lens and also changed the distance between the light source and the cell. The manner in which the crystals emerged was then assessed while adjusting the irradiation time such that the number of photons irradiating the cell was held constant at $1.5 \times 10^{17}$. As a control, we performed an additional trial in which the sample was not irradiated. Figure 4 shows the results of the crystallization experiments, including the control performed without irradiation and the trials in which the light fluence was varied by a factor of nine. The results indicate that crystallization is enhanced when applying a low fluence compared to the control solution without irradiation. Crystallization is suppressed at high fluence, under which conditions the combination of the initial Trp ${ }^{62}$ radicals readily occurs. For this reason, it is thought that the $\operatorname{Tr} p^{62}-\operatorname{Trp}^{62}$ dimers formed under high fluence do not act as templates for crystal nucleation.

The results of these trials suggest that templates are readily generated under a low fluence. Under these conditions, the radical concentration is low and a mechanism in which the concentration of Trp radicals decays via a 
first order process to yield Tyr radicals proceeds preferentially. The subsequent collision of these radicals with ground state protein molecules to generate dimers is believed to occur under these circumstances. From these results, we can draw some conclusions regarding the nature of the reactions that proceed under irradiation.

Based on the above data, we propose a mechanism in which Tyr radicals on one protein react with Tyr residues on another protein to form dimers, as illustrated in Scheme 2(c). The formation of dimers between Tyr residues is predicated on fluorescence data specific to this combination [22]. Figure 5 shows the results of trials in which we used fluorescence spectra to confirm the formation of a dimer between two Tyr residuals following a photochemical reaction. Figure 5(a) presents the fluorescence spectra obtained when the amino acid Tyr, rather than the complete protein, was exposed to UV radiation as a control experiment. The Tyr absorption and fluorescence spectra are indicated by solid and dotted lines, respectively. The fluorescence maximum appears at $310 \mathrm{~nm}$ in the case of the original sample, but irradiation shifts this maximum to $430 \mathrm{~nm}$. This change is attributed to the emission of the Tyr-Tyr dimer. Figure 5(b) shows the change in the fluorescence spectrum of the lysozyme after exposure to UV light. The absorption spectrum of the lysozyme is shown as a solid line and its fluorescence spectrum is shown as a dotted line. Irradiation of the lysozyme generates a fluorescence peak in approximately the same wavelength region as the fluorescence peak of the Tyr-Tyr dimer, as shown in Figure 5(a). This experiment, therefore, strongly supports a mechanism 
in which protein dimers of Tyr residues are generated by photochemical reaction of the lysozyme.

\subsection{Structure of the dimer and template}

The lysozyme has only three surface sites at which Tyr residues can form dimers. Because the positions where Tyr residues can form dimers are limited, the number of possible dimers is also restricted. Specifically, Tyr residues are situated at the 20th, 23rd and 53rd sites on the surface of the lysozyme. The dimer structures that can be formed from combinations of these are restricted to six types: dimers formed by the combination of three types of homogeneous residues $\left(\mathrm{Tyr}^{20}-\mathrm{Tyr}^{20}, \mathrm{Tyr}^{23}-\mathrm{Tyr}^{23}\right.$ and $\left.\mathrm{Tyr}{ }^{53}-\mathrm{Tyr}^{53}\right)$ and dimers formed by the combination of heterogeneous residues $\left(\mathrm{Tyr}^{20}\right.$ $\mathrm{Tyr}^{23}$, Tyr ${ }^{20-T y r}{ }^{53}$ and Tyr $\left.{ }^{23-T y r}{ }^{53}\right)$. Figure 6 shows the six types of dimer structures that can be formed with these amino acids.

To confirm which of the six possible dimers can function as templates, we compared these dimers to the alignment of two adjacent molecules in the crystal. Figure 7 shows the packing of molecules in the lysozyme unit cell [23], with the Tyr ${ }^{53}$ residues in red. It is noteworthy that the Tyr ${ }^{53}$ residue in the blue molecule in the center of the structure and the $\mathrm{Tyr}^{53}$ residue in the other blue molecule are aligned face-to-face. This structure is similar to the dimers formed by the combination of $\mathrm{Tyr}^{53}-\mathrm{Tyr}^{53}$ shown in Figure 6 . Therefore, we suggest that Tyr ${ }^{53-T y r^{53}}$ dimers serve as templates for crystal growth. 


\section{Conclusions}

We have demonstrated that dimers having the same alignment as the two adjacent molecules in a crystal, formed via the photochemical reaction of a lysozyme, can act as templates that grow into crystal nuclei. The excited lysozyme first reacts to form Trp radicals as reaction intermediates which subsequently combine to give Trp-Trp linked dimers. These, however, do not act as templates. Trp radicals react in the lysozyme molecule, leading to the formation of Tyr radicals that again can dimerize. Six possible Tyr-Tyr dimers are proposed, of which the Tyr ${ }^{53}-\mathrm{Tyr}^{53}$ combination exhibits an alignment similar to the two adjacent molecules in the crystal and thus is thought to behave as a template.

\section{Acknowledgements}

This study was conducted as a part of the PRESTO project "Innovative use of light and materials/life" funded by the Strategic Basic Research Programs of the National Institute of the Japan Science and Technology Agency. 


\section{References}

[1] B. A. Garetz, J. E. Aber, N. L. Goddard, R. G. Young, and A. S. Myerson, Nonphotochemical, polarization-dependent, laser-induced nucleation in supersaturated aqueous urea solutions, Physical Review Letters, 77, (1996) 3475 http://dx.doi.org/10.1103/PhysRevLett.77.3475

[2] J. Zaccaro, J. Matec, A. S. Myerson, and B. A. Garetz, Nonphotochemical, laserinduced nucleation of supersaturated aqueous glycine produces unexpected $\gamma$ polymorph, Cryst. Growth Des., 1, (2001) 5-8. DOI: 10.1021/cg0055171

[3] B. A. Garetz, J. Matic, and A. S. Myerson, Polarization switching of crystal structure in the nonphotochemical light-induced nucleation of supersaturated aqueous glycine solutions, Phys. Rev. Lett., 89 (2002) 175501. http://dx.doi.org/10.1103/PhysRevLett.89.175501

[4] X. Sun and B. A. Garetz, Supersaturation and polarization dependence of polymorph control in the nonphotochemical laser-induced nucleation (NPLIN) of aqueous glycine solutions, Cryst. Growth Des., 6 (2006) 684-689. DOI: $10.1021 / \operatorname{cg} 050460+$

[5] H. Adachi, K. Takano, Y. Hosokawa, T. Inoue, Y. Mori, H. Matsumura, M. Yoshimura, Y. Tsunaka, M. Morikawa, S. Kanaya, H. Masuhara, Y. Kai, and T. Sasaki, Laser irradiated growth of protein crystal, Jpn. J. Appl. Phys., 42 (2003) L798-L800. DOI:10.1143/JJAP.42.L798

[6] H. Yoshikawa, R. Murai, S. Maki, T. Kitatani, S. Sugiyama, G. Sazaki, H. Adachi, T. Inoue, H. Matsumura, K. Takano, S. Murakami, T. Sasaki, and Y. Mori, Laser energy dependence on femtosecond laser-induced nucleation of protein, Appl. Phys. A, 93 (2008) 911-915. DOI:10.1007/s00339-008-4790-x 
[7] H. Y. Yoshikawa, R. Murai, S. Sugiyama, G. Sazaki, T. Kitatani, Y. Takahashi, H. Adachi, H. Matsumura, S. Murakami, T. Inoue, K. Takano, and Y. Mori, Femtosecond laser-induced nucleation of protein in agarose gel crystals, J. Cryst. Growth, 311 (2009) 956-959. DOI:10.1016/j.jcrysgro.2008.09.137

[8] H. Y. Yoshikawa, Y. Hosokawa, and H. Masuhara, Explosive crystallization of urea triggered by focused femtosecond laser irradiation, Jpn. J. Appl. Phys., 45 (2006) L23-L26. DOI:10.1143/JJAP.45.L23

[9] K. Nakamura, Y. Hosokawa, and H. Masuhara, Anthracene crystallization induced by single-shot femtosecond laser irradiation: experimental evidence for the important role of bubbles, Cryst. Growth Des., 7 (2007) 885-889. DOI: $10.1021 / \operatorname{cg} 060631 \mathrm{q}$

[10]T. Sugiyama, T. Adachi, and H. Masuhara, Crystallization of glycine by photon pressure of a focused CW laser beam, Chem. Lett., 36 (2007) 1480-1481. DOI: $10.1246 / \mathrm{cl} .2007 .1480$

[11]T. Okutsu, T. Terao, H. Hiratsuka, A. Yamano, N. Ferté, and S. Veesler, Lightinduced nucleation of metastable hen egg-white lysozyme solutions, Cryst. Growth Des., 5 (2005) 1393. DOI:10.1021/cg049604v

[12]S. Veesler, K. Furuta, H. Horiuchi, H. Hiratsuka, N. Ferté, and T. Okutsu, Crystals from light: photochemically induced nucleation of hen egg-white lysozyme, Cryst. Growth Des., 6 (2006) 1631-1635. DOI:10.1021/cg0506424

[13]T. Okutsu, Photochemically-induced crystallization of protein, J. Photochem. Photobiology C: Photochemistry Reviews, 8 (2007) 143-155.

DOI:10.1016/j.jphotochemrev.2007.06.002

[14]T. Okutsu, M. Sato, K. Furuta, Y. Fujinaga, K. Horota, K. Obi, H. Horiuchi, and H. 
Hiratsuka, Enhancement of light-induced nucleation of lysozyme in the presence of polyethylene glycol (PEG) 4000, Chem. Lett., 36 (2007) 338-339. http://doi.org/10.1246/cl.2007.338

[15]T. Okutsu, K. Sugiyama, K. Furuta, I. Watanabe, H. Mori, K. Obi, K. Horota, H. Horiuchi, G. Sazaki, S. Veesler, and H. Hiratsuka, Photochemically induced nucleation in supersaturated and undersaturated thaumatin solutions, J. Photochem. Photobiol. A: Chemistry, 190 (2007) 88-93. DOI:10.1016/j.jphotochem.2007.03.016

[16]K. Furuta, T. Okutsu, G. Sazaki, I. Yoshizaki, H. Horiuchi, T. Shimizu, M. Yamamoto, Y. Tanaka, and H. Hiratsuka, Detection of covalent-bonded dimer in photochemically induced crystallization of protein, Chem. Lett., 36 (2007) 714-715. http://doi.org/10.1246/c1.2007.714

[17]K. Furuta, H. Horiuchi, H. Hiratsuka, and T. Okutsu, Photochemically induced nucleation of ribonuclease A enhanced by a stable protein dimer produced from the photochemical reaction of Tyr residual groups, Cryst. Growth Des., 8 (2008) 18861889. DOI:10.1021/cg701016p

[18]A. McPherson, Preparation and Analysis of Protein Crystals, Krieger Publishing Company, Malabar, FL, 1982.

[19]D. E. McRee, Practical Protein Crystallization, second ed., Academic Press, San Diego, CA, 1993.

[20]L. I. Grossweiner, A. G.Kaluskar, and J. F. Baugher, Flash photolysis of enzymes, Int. J. Radiat. Biol. 29 (1976) 1-16. DOI:10.1080/09553007614551511

[21]L. I. Grossweiner and Y. Usui, Flash photolysis and inactivation of aqueous lysozyme, Photochem. Photobilol. 13 (1971) 195-214. DOI: 10.1111/j.17511097.1971.tb06106.x 
[22] S. Hashimoto, A. Kira, M. Imamura, and T. Masuda, Lysozyme dimer formation on lysozyme oxidation with $\mathrm{Br}_{2}$ radical as studied by fluorescence evolution, Int. J.

Radiat. Biol., 41 (1982) 303-314. DOI:10.1080/09553008214551761

[23]R. Diamond, D. C. Phillips, C. C. F. Blake, and A. C. T. North, Real-space refinement of the structure of hen egg-white lysozyme, J. Mol. Biol., 82 (1974) 371391. DOI: $10.1016 / 0022-2836(74) 90598-1$ 


\section{Figure Captions}

Figure 1. Photographic images of protein solutions: (a) a non-irradiated control, (b) an irradiated solution, (c) a solution with pre-irradiated crystalline lysozyme.

Figure 2. SDS-PAGE results for the photo-irradiated lysozyme. Right: nonirradiated control solution, in which no dimer band is observed. Centre: irradiated solution. Left: solution of a pre-irradiated crystalline sample, in which the dimer band is observed more clearly than in the irradiated solution sample.

Figure 3. (a) Transient absorption spectra of the lysozyme acquired 50 and $400 \mu \mathrm{s}$ after laser excitation, (b) decay profile of the intermediate lysozyme radical as observed at $550 \mathrm{~nm}$ when applying excitation laser fluence values of 7 and $2 \mathrm{~mJ}$ pulse ${ }^{-1}$, (c) reciprocal decay profile at high fluence, and (d) logarithmic decay profile at high and low fluence values.

Figure 4. Average numbers of crystals appearing in a droplet at various values of irradiation photon fluence. The left bar indicates the control experiment without irradiation.

Figure 5. Absorption (solid line), fluorescence (dotted line) and emission (dotted line) spectra after irradiation of (a) Tyr and (b) the lysozyme. The emissions above $350 \mathrm{~nm}$ correspond to that from the Tyr-Tyr chromophore. 
Figure 6. Configurations of dimers of Tyr-Tyr residues that may be produced by photochemical reactions.

Figure 7. Molecular packing of the lysozyme molecules within the unit cell. 
Figure 1

(a)

(b)

(c)
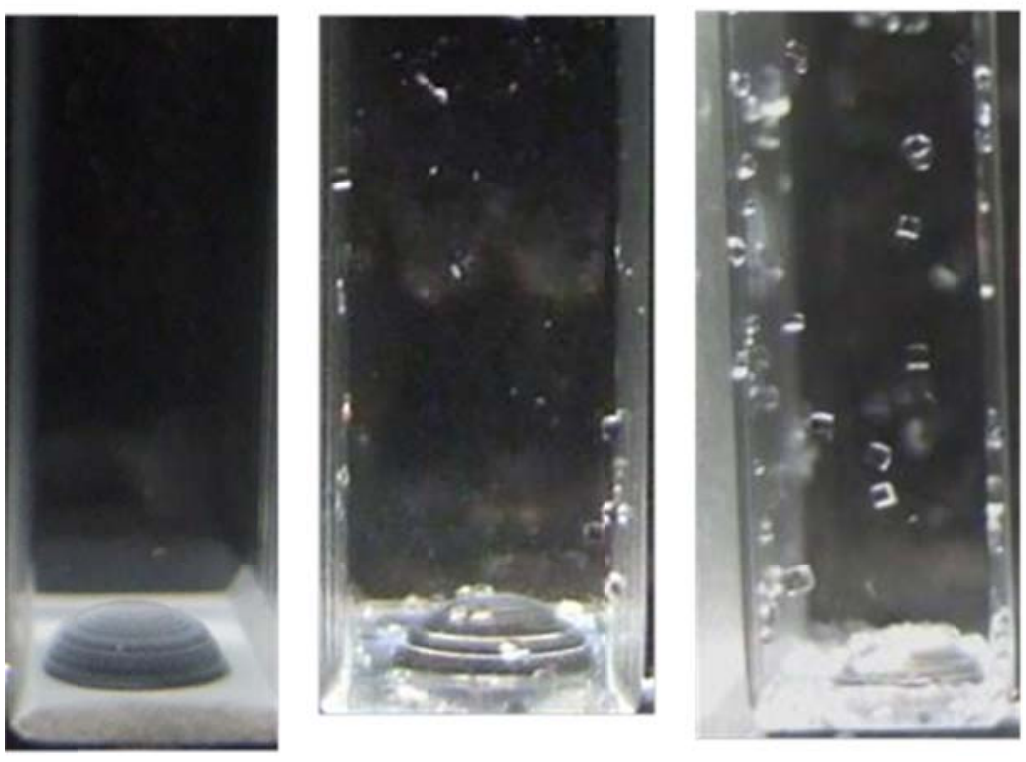
Figure 2

Marker UV- UV+ UV+ solution crystal

Dimer

Monomer 
Figure 3
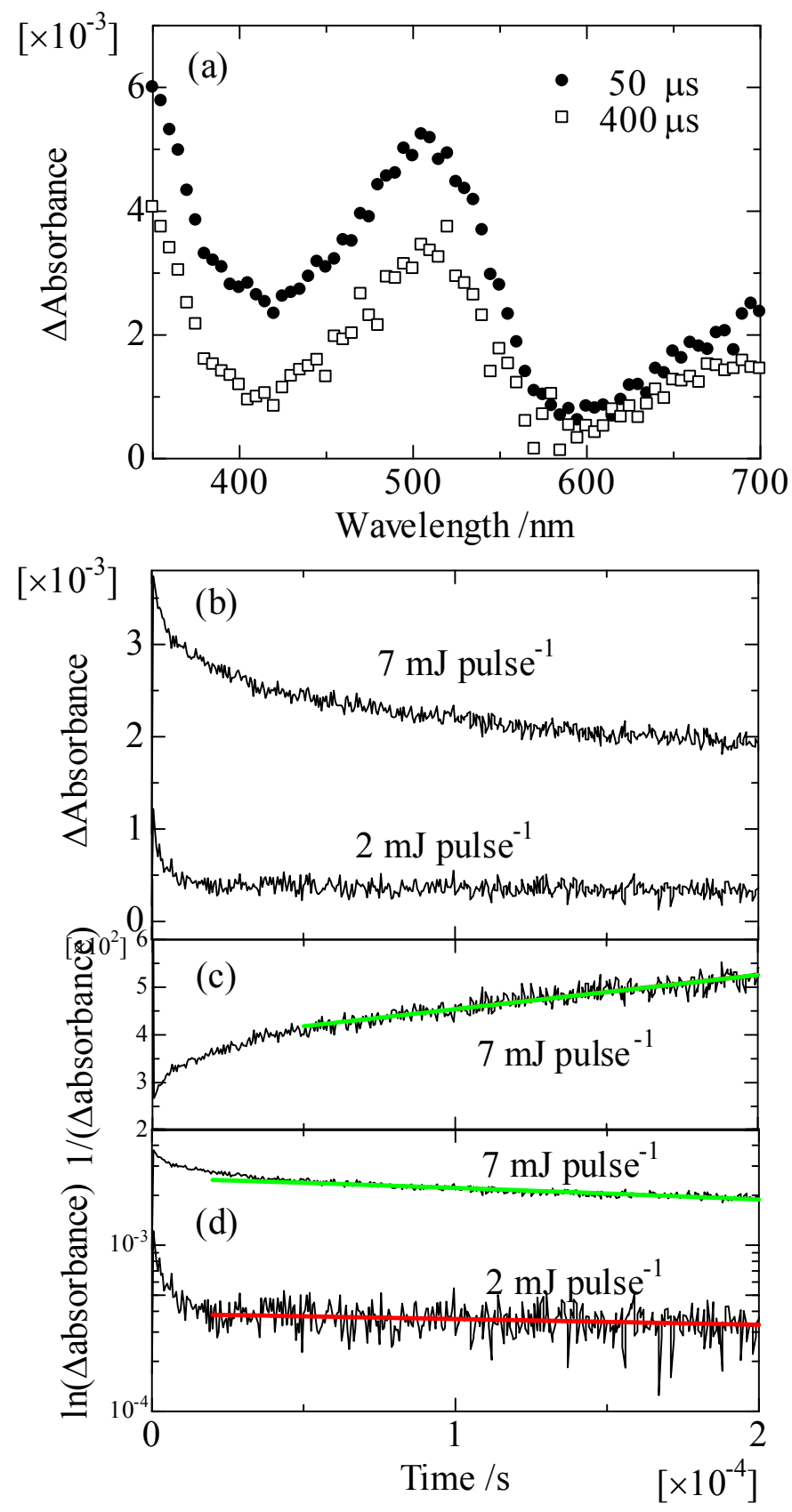
Figure 4

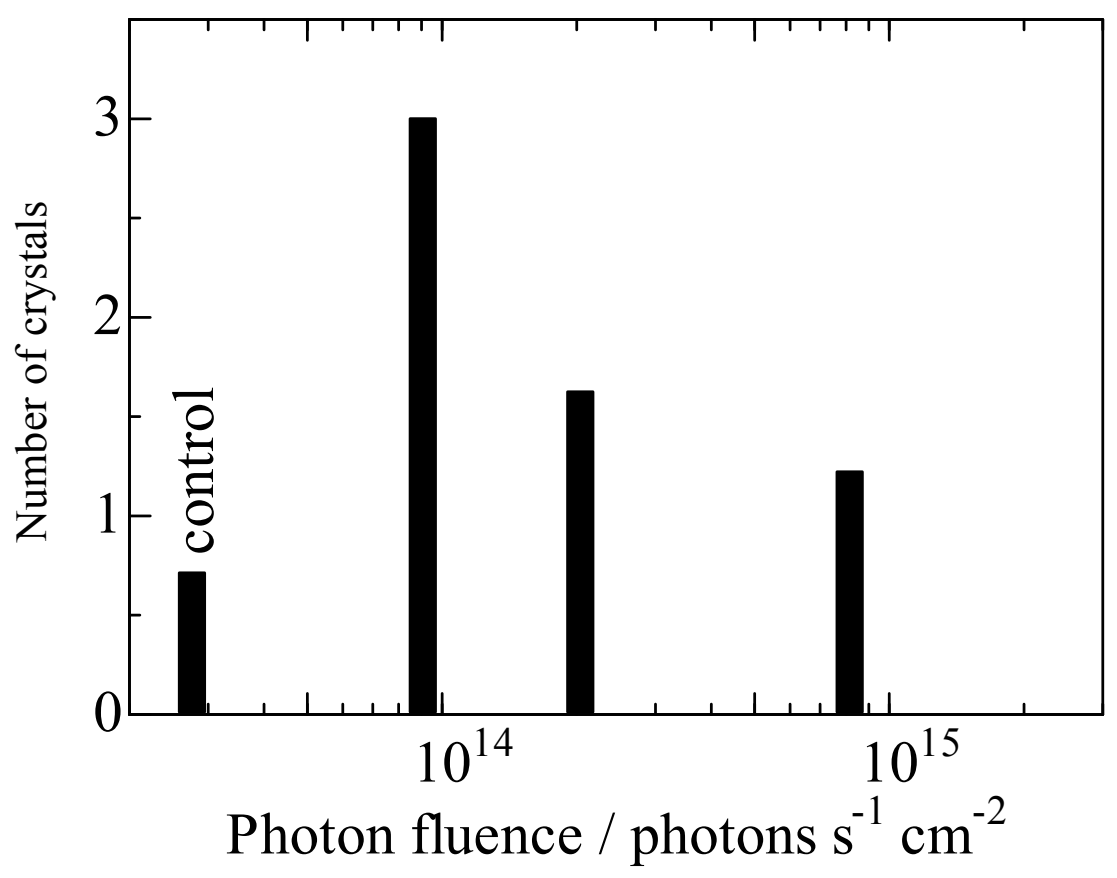


Figure 5

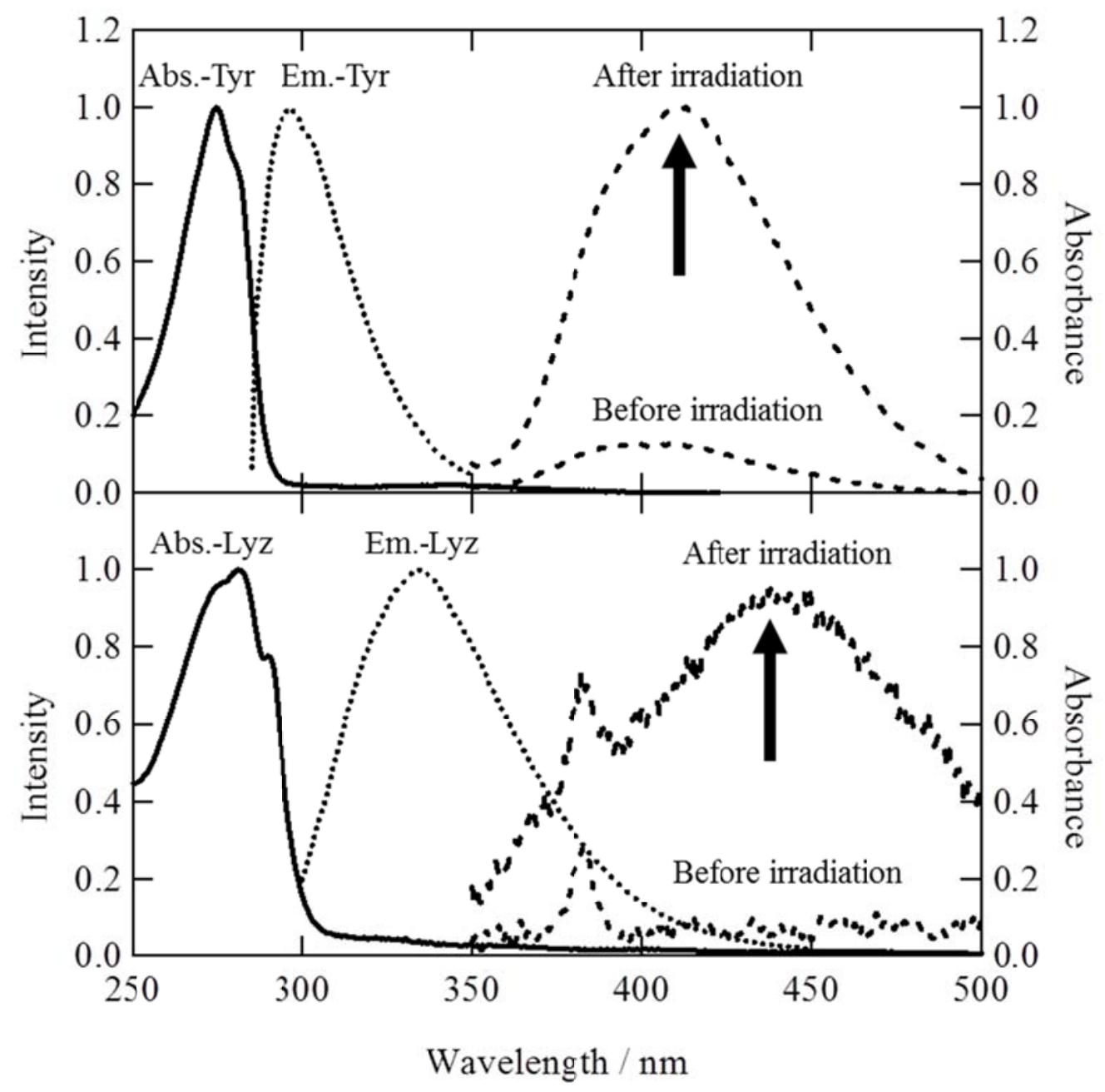


Figure 6
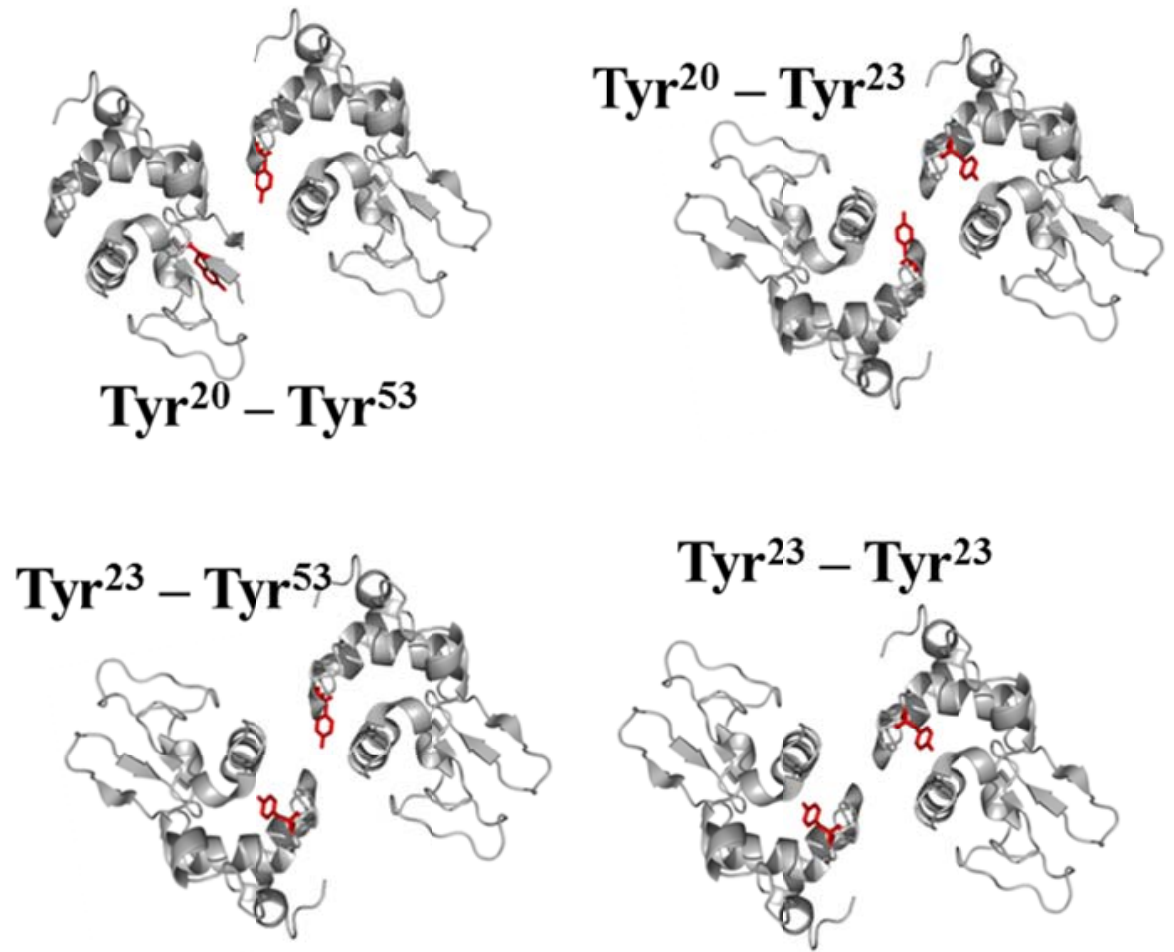

$\mathrm{Tyr}^{23}-\mathrm{Tyr}^{23}$
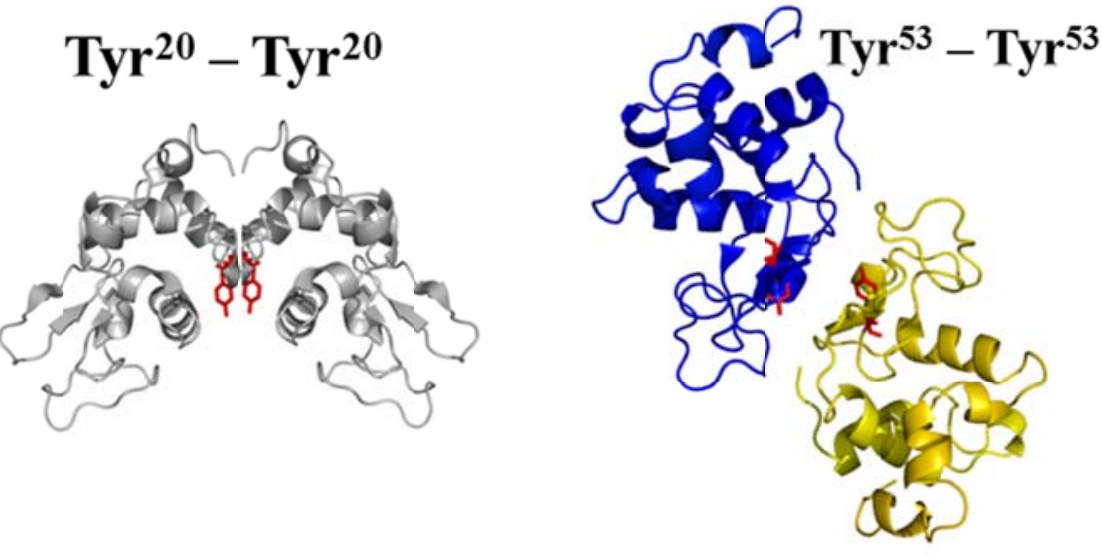
Figure 7

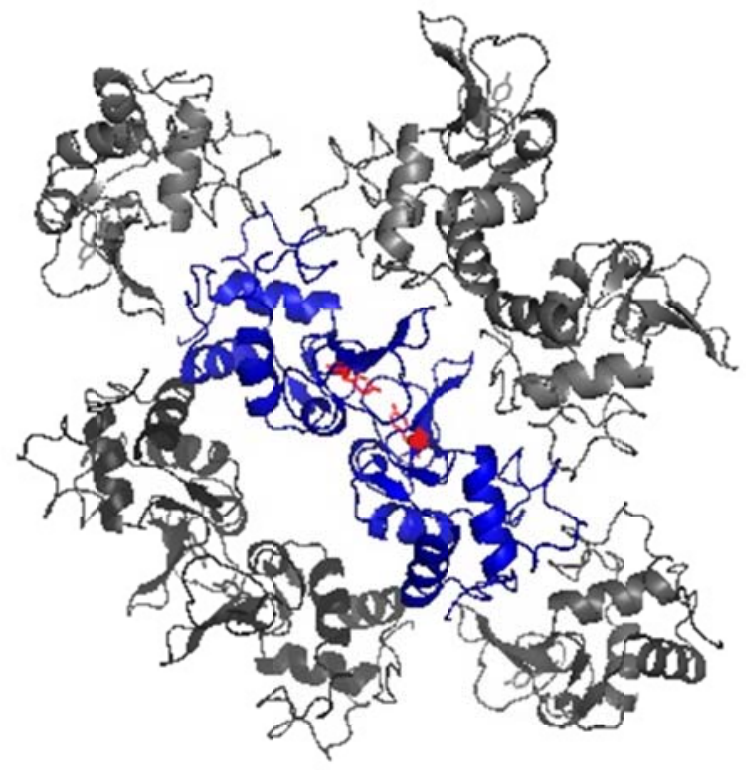


Scheme 1

(a)

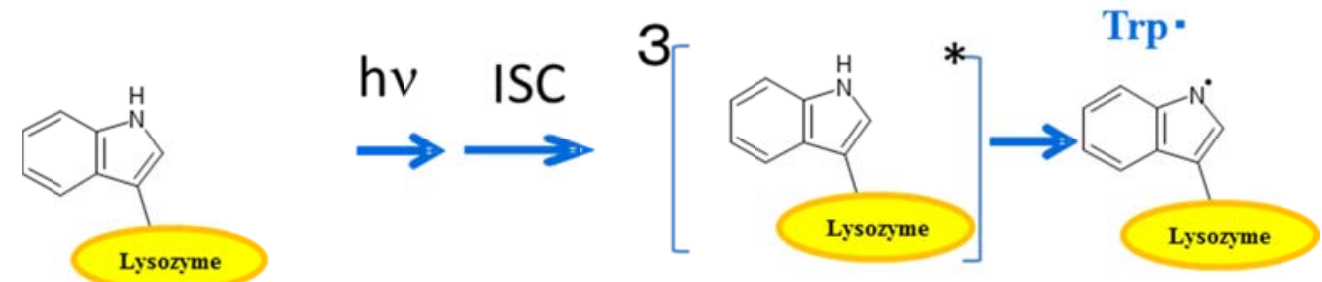

(b)
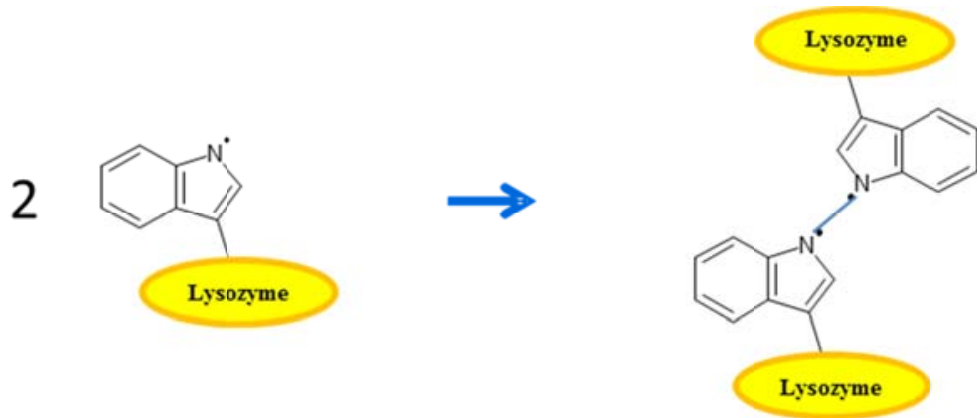

(c)

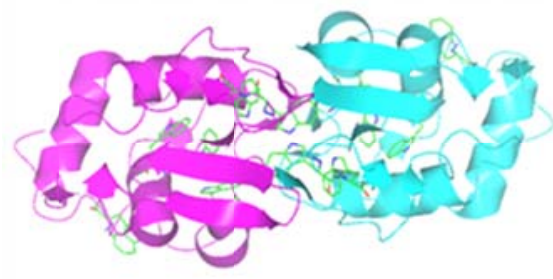

(d)
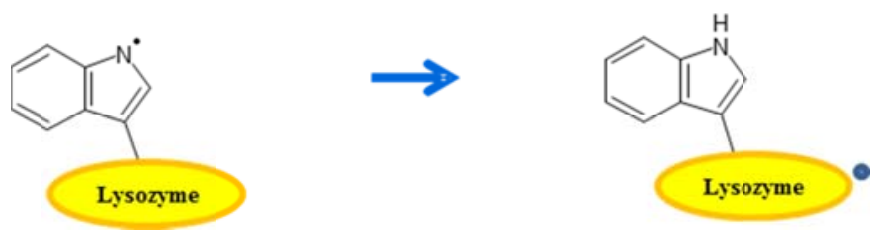
Scheme 2

(a)

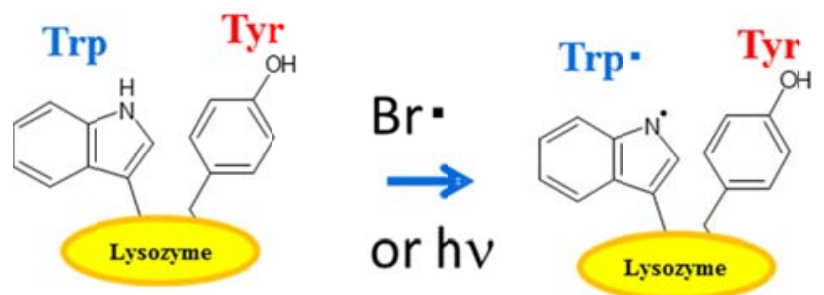

(b)
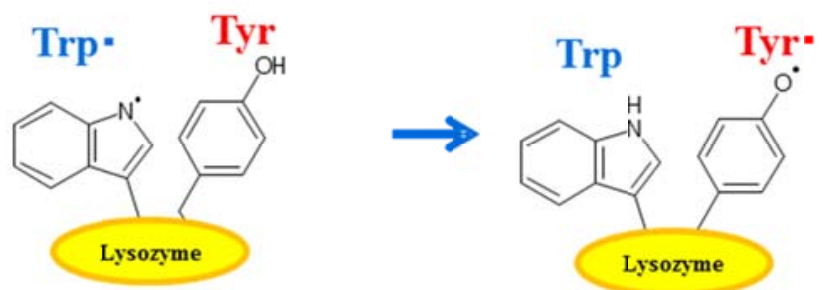

(c)
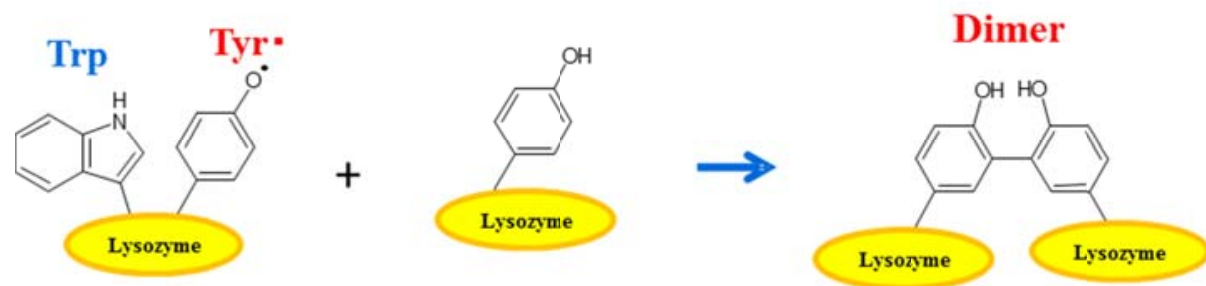
Graphical Abstract

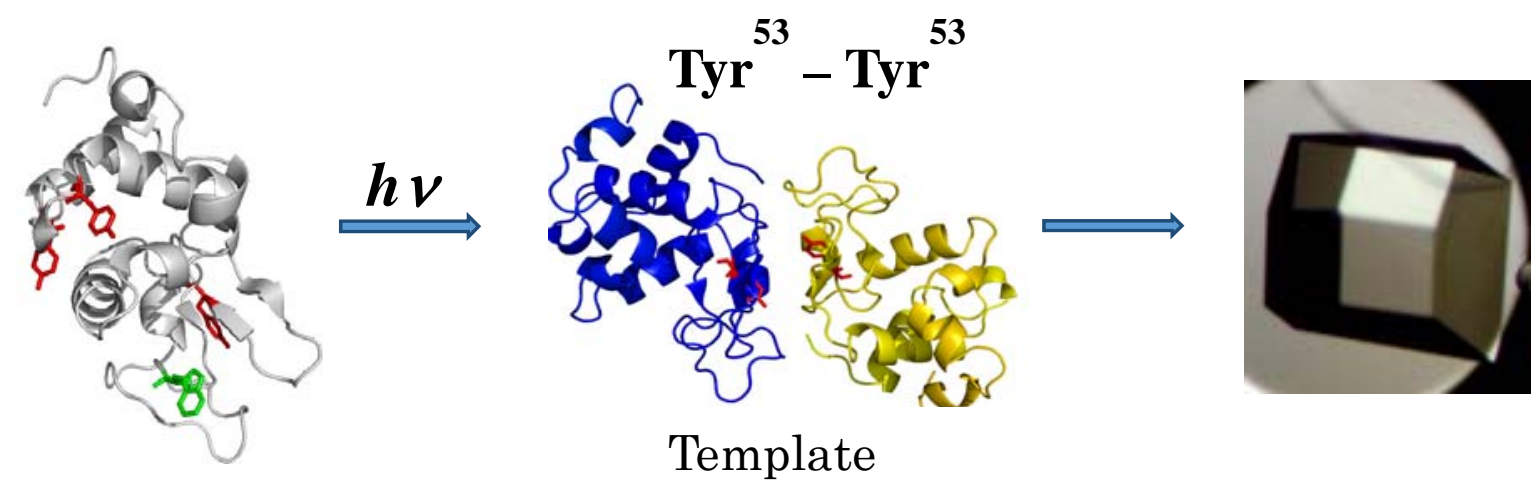

